\title{
Diagnostic accuracy of pleural fluid NT-pro-BNP for pleural effusions of cardiac origin: a systematic review and meta-analysis
}

\author{
Surinder Janda, John Swiston*
}

\begin{abstract}
Background: Several studies have been published in the literature on the diagnostic accuracy of NT-pro-BNP for pleural effusions from heart failure in the last decade. The purpose of our study was to perform a systematic review and meta-analysis on the diagnostic accuracy of pleural fluid NT-pro-BNP for pleural effusions of cardiac origin.

Methods: MEDLINE, EMBASE, PapersFirst, and the Cochrane collaboration and the Cochrane Register of controlled trials were searched. All searches were inclusive as of March 2010. Studies were only included if the absolute number of true-positive, false-negative, true-negative, and false-positive observations were available, and the "reference standards" were described clearly. Two investigators independently reviewed articles and extracted data. Quality was assessed with the Quality Assessment for Diagnostic Accuracy Studies (QUADAS). The bivariate model for diagnostic meta-analysis was used to obtain a pooled sensitivity and a pooled specificity.

Results: Ten studies (total number of patients 1120) were included in the meta-analysis. The average pleural fluid NT-pro-BNP level in effusions of cardiac origin was $6140 \mathrm{pg} / \mathrm{mL}$. The pooled sensitivity and specificity of all studies combined was 94\% (95\% Cl: 90-97) and 94\% (95\% Cl: 89-97) respectively. The pooled positive likelihood ratio was 15.2 (95\% Cl: 8.1-28.7) and the pooled negative likelihood ratio was 0.06 (95\% Cl: $0.03-0.11)$. The area under the ROC curve was 0.98 (95\% Cl: 0.96-0.99) and the diagnostic odds ratio was 246 (95\% Cl: 81-745).
\end{abstract}

Conclusions: Pleural fluid NT-pro-BNP is a very useful biomarker with high diagnostic accuracy for distinguishing pleural effusions of cardiac origin.

\section{Background}

Pleural effusions arising from heart failure are usually discriminated from other causes based on clinical criteria in association with biochemical analysis, particularly the discrimination of transudates versus exudates, most commonly using Light's criteria [1]. The sensitivity of Light's criteria for identifying exudative pleural effusions is very high (98\%)[2], however the criteria's ability to exclude transudative effusions is low[3]. As a result, heart failure associated pleural effusions can be misclassified as exudates using Light's criteria, particularly after diuretics have been used. One study showed that $28 \%$ (5 of 18 ) patients with pleural effusions from congestive heart

\footnotetext{
* Correspondence: swiston@interchange.ubc.ca
Division of Respiratory Medicine, The University of British Columbia,

* Correspondence: swiston@interchange.ubc.ca Vancouver, BC, Canada
}

(c) 2010 Janda and Swiston; licensee BioMed Central Ltd. This is an Open Access article distributed under the terms of the Creative Commons Attribution License (http://creativecommons.org/licenses/by/2.0), which permits unrestricted use, distribution, and reproduction in any medium, provided the original work is properly cited.

failure (CHF) were misclassified as exudative effusions using Light's criteria[4]. A diagnostic dilemma often arises when CHF-associated pleural effusions are misclassified as exudates which can then lead to the use of more expensive and sometimes invasive tests to diagnose the etiology of the effusion. A non-invasive and inexpensive strategy to discriminate pleural effusions of a cardiac origin would be beneficial in such circumstances.

Serum brain natriuretic peptide (BNP) or the aminoterminal fragment $\mathrm{N}$-terminal pro-brain natriuretic peptide (NT-pro-BNP) have an established role in the diagnosis, management, and prognosis of patients with CHF[5]. BNP, also known as B-type natriuretic peptide, is a 32 amino acid polypeptide secreted by the ventricles of the heart in response to excessive stretching of cardiomyocytes[6]. BNP is co-secreted along with a 76 amino acid polypeptide, NT-pro-BNP, which is biologically 
inactive[7]. The half-life of BNP is approximately 20 minutes whereas of NT-pro-BNP is 1-2 hours[8]. BNP binds to atrial natriuretic factor receptors leading to a decrease in systemic vascular resistance and central venous pressure, and an increase in natriuresis[6].

Several studies have been published in the literature on the diagnostic accuracy of NT-pro-BNP for pleural effusions from heart failure. The purpose of our study was to perform a systematic review and meta-analysis on the diagnostic accuracy of pleural fluid NT-pro-BNP for pleural effusions of cardiac origin.

\section{Methods}

The systematic review and meta-analysis was performed according to the recently published recommendations and checklist of the PRISMA statement[9].

Searches were conducted on MEDLINE (inception-March 2010); EMBASE (inception-March 2010), PapersFirst (inception-March 2010), and the Cochrane collaboration and the Cochrane Register of controlled trials for relevant studies. The following key terms were used: 'pleural fluid' or 'pleural effusion' AND 'brain natriuretic peptide' or 'B-type natriuretic peptide' or 'BNP', 'pro-BNP' or 'NT-pro-BNP' or 'aminoterminal pro-BNP' or 'congestive heart failure' or 'heart failure' or 'CHF'. All searches were limited to 'humans'. We identified additional studies by searching the bibliographies of retrieved articles. Two independent reviewers (SJ and JS) performed the literature search. Studies relevant to the diagnostic value of NT-pro-BNP for pleural effusions of cardiac origin were included if the following criteria were met: pleural fluid NT-pro-BNP was used for diagnosing pleural effusions of cardiac origin; and a $2 \times 2$ contingency table could be formulated from the available data. Studies that used BNP or other biomarkers were excluded.

All studies that appeared to fit the inclusion criteria were identified for full review by two reviewers (SJ and JS). Each reviewer independently selected studies for inclusion in the review. Disagreement between the two extracting authors was resolved by consensus.

The methodological quality of the selected studies was graded independently by two reviewers (SJ and JS) with the Quality Assessment of Diagnostic Accuracy Studies (QUADAS) tool, a validated tool for the quality assessment of diagnostic accuracy studies[10]. We performed component analysis using the QUADAS tool which was depicted as a proportional bar graph for each of the 14 individual criteria. Disagreement between the two extracting authors was resolved by consensus.

The following variables were extracted from each study: publication year; country of origin of study; study design; patient demographics and co-morbidities; NT-pro-BNP assay type; numbers of true-positive, falsenegative, true-negative, and false-positive observations; correlation statistic between pleural fluid and serum
NT-pro-BNP if available; and the reference standard used.

We used the bivariate model for diagnostic metaanalysis to obtain an overall sensitivity and an overall specificity[11]. Instead of using the diagnostic odds ratio, as used in conventional diagnostic meta-analysis [12], the bivariate model uses pairs of sensitivity and specificity as the starting point of the analysis. In addition to accounting for study size, the bivariate model estimates and incorporates the negative correlation that may arise between the sensitivity and specificity of the index test within studies as a result of differences in test threshold between studies. The bivariate model uses a random effects approach for both sensitivity and specificity, which allows for heterogeneity beyond chance as a result of clinical and methodological differences between studies. The pooled estimates of sensitivity and specificity were used to calculate the average positive and negative likelihood ratios. Publication bias through small study effects was assessed with a regression test on the diagnostic odds ratio[13].

A receiver operating characteristic graph, with the $y$-axis representing the index test's sensitivity (true positive rate) and the $x$-axis representing 1-specificity (false negative rate), was used to plot the individual and summary points of sensitivity and specificity. Furthermore, around the pooled estimates, we also plotted a $95 \%$ confidence region and a $95 \%$ prediction region to illustrate the precision with which the pooled values were estimated (confidence ellipse of a mean) and to show the amount of between study variation (prediction ellipse; the likely range of values for a new study). We used Stata intercooled version 10.1 (StataCorp, College Station, Texas), in particular the midas and metandi commands, for all statistical analyses[14,15].

\section{Results}

Our search yielded 128 citations of which 117 were excluded for various reasons based on the title and abstract (Figure 1). Eleven were then retrieved for full text review of which three were excluded because BNP instead of NT-pro-BNP was used as the biomarker (Figure 1). Ten studies were included in the final analysis [16-25]. One study, by Seyhan et al[22], was initially published online and then was retracted by the editors of the journal. The reason for retraction was due to a violation of the journal's Information for Authors. Therefore, only the abstract was available for data extraction and a quality assessment was not possible.

The studies were published from 2004 to 2010. The majority of the studies (7/9)[16-19,22-24] were done in Europe whereas two were done in North America[20,25] and one in Asia[21]. All studies except for two (Porcel et el[23] and Long et al[25]) were of prospective design 


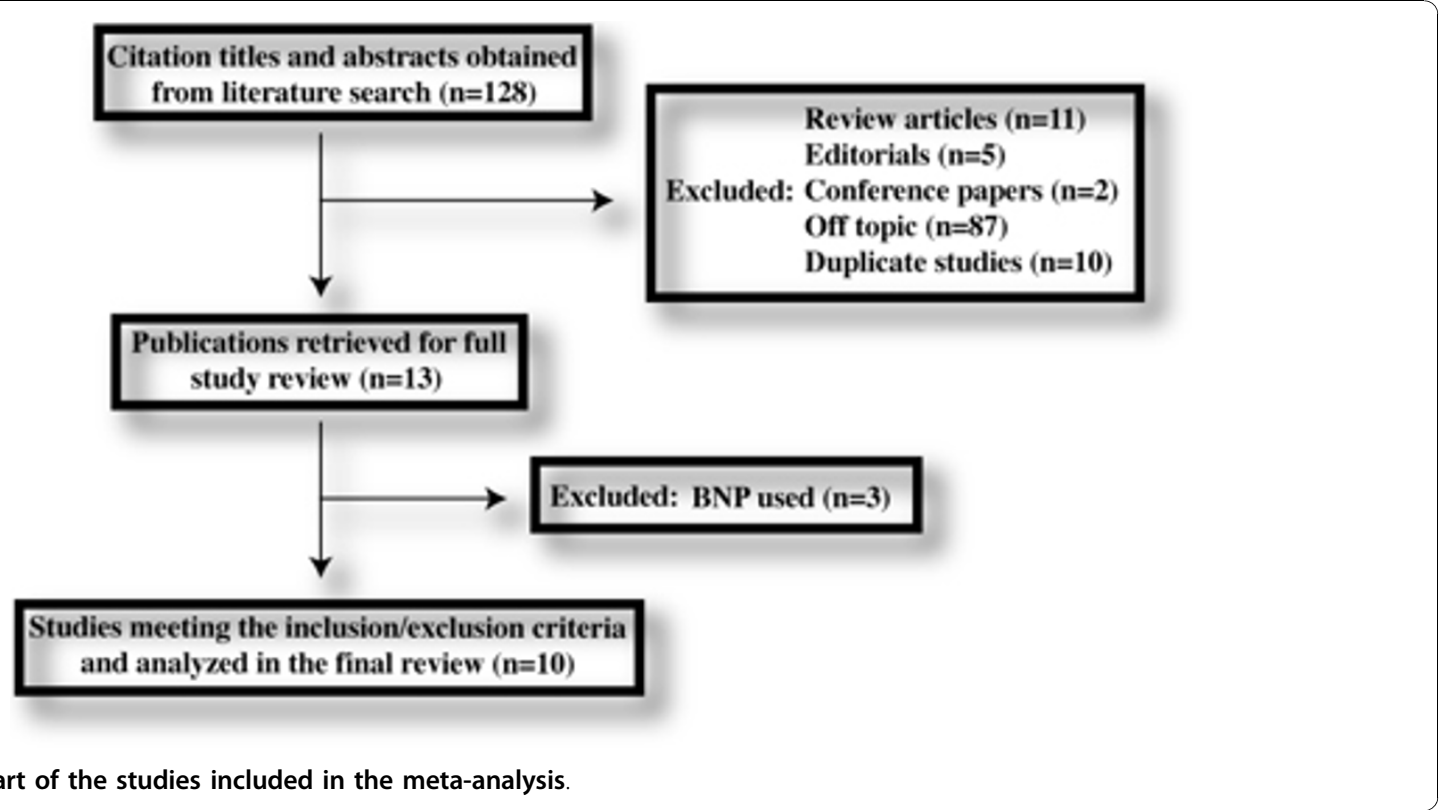

(Table 1). Furthermore, all studies except for two (Liao et al[20] and Long et al[25]) used an electrochemical luminescence immunoassay (ELCIA) performed on the Elecsys 2010 analyzer (Roche) to measure levels of NTpro-BNP whereas Liao et al[20] and Long et al[25] used an enzyme-linked immunosorbent assay (ELISA) (Table 1). A total of 1120 patients/pleural effusions were included in this analysis (429 of cardiac origin and 691 of non-cardiac origin). The control group (non-cardiac pleural effusions) consisted of mainly malignant pleural effusions and infections (Table 1).

As there is no gold standard for effusions of cardiac origin, clinical criteria was used as the reference standard. All studies used some combination of the following criteria: history, physical exam, chest $x$-ray, electrocardiogram, echocardiogram, and response to

Table 1 Characteristics of the studies included in the meta-analysis.

\begin{tabular}{|c|c|c|c|c|c|c|}
\hline Study & Year & Country & Design & Assay Type & Reference Standard & Control Group $^{\dagger}$ \\
\hline Tomcsanyi et al [16] & 2004 & Hungary & PC & $\mathrm{ECLIA}(1)^{\ddagger}$ & $\begin{array}{c}\text { Clinical Criteria* } \\
a, b, c, e\end{array}$ & $57 \%$ M; $14 \%$ l; 29\% O \\
\hline Porcel et al [17] & 2004 & Spain & PC & ECLIA(1) & $\begin{array}{c}\text { Clinical Criteria* } \\
\text { a, b, c, d, e,f }\end{array}$ & NR \\
\hline Kolditz et al [18] & 2006 & Germany & PC & $\mathrm{ECLIA}(1)$ & $\begin{array}{c}\text { Clinical Criteria* } \\
\text { a, b, c, e, f }\end{array}$ & $59 \%$ M; 21\% l; 30\% O \\
\hline Porcel et al [19] & 2007 & Spain & PC & $\mathrm{ECLIA}(1)$ & $\begin{array}{c}\text { Clinical Criteria* } \\
a, b, c, d, e, f\end{array}$ & $48 \%$ M; $20 \%$ l; $32 \% \mathrm{O}$ \\
\hline Liao et al [20] & 2008 & USA & PC & ELISA & $\begin{array}{c}\text { Clinical Criteria* } \\
a, c, e\end{array}$ & $25 \% \mathrm{M} ; 0 \% \mathrm{l} ; 75 \% \mathrm{O}$ \\
\hline Han et al [21] & 2008 & Korea & PC & ECLIA(1) & $\begin{array}{c}\text { Clinical Criteria* } \\
a, b, e, f\end{array}$ & $24 \% \mathrm{M} ; 66 \% \mathrm{l} ; 10 \% \mathrm{O}$ \\
\hline Seyhan et al [22] & 2009 & Turkey & PC & ECLIA & $\begin{array}{c}\text { Clinical Criteria* } \\
\text { N/A }\end{array}$ & $\mathrm{N} / \mathrm{A}$ \\
\hline Porcel et al [23] & 2009 & Spain & $\mathrm{RC}$ & ECLIA(2) & $\begin{array}{c}\text { Clinical Criteria* } \\
\text { a, b, c, e, } f\end{array}$ & $44 \%$ M; $29 \%$ I; $27 \%$ \\
\hline Bayram et al [24] & 2009 & Turkey & PC & ECLIA(1) & $\begin{array}{c}\text { Clinical Criteria* } \\
\text { a, b, c, e, } f\end{array}$ & $23 \% \mathrm{M} ; 31 \% \mathrm{l} ; 46 \% \mathrm{O}$ \\
\hline Long et al [25] & 2010 & USA & $\mathrm{RC}$ & ELISA & $\begin{array}{c}\text { Clinical Criteria* } \\
\text { a, b, c, e }\end{array}$ & $25 \% \mathrm{M} ; 25 \% \mathrm{l} ; 25 \%$ \\
\hline
\end{tabular}

* = heart failure diagnosis based on (a) history, or (b) physical exam, or (c) chest x-ray, or (d) electrocardiogram, or (e) echocardiogram, or (f) response to diuretics; $\dagger=$ non-cardiac effusions; $\neq=$ generation of assay (one or two); ECLIA = electrochemical luminescence immunoassay; ELISA = enzyme-linked immunosorbent assay; I = infectious effusion; $M=$ malignant effusion; N/A = not available; $N R=$ not recorded; $O=$ other causes (i.e. connective tissue disease, pleuritis, post-CABG, hepatic hydrothorax, pulmonary embolism, etc); $\mathrm{PC}=$ prospective cohort; $\mathrm{RC}=$ retrospective cohort; 


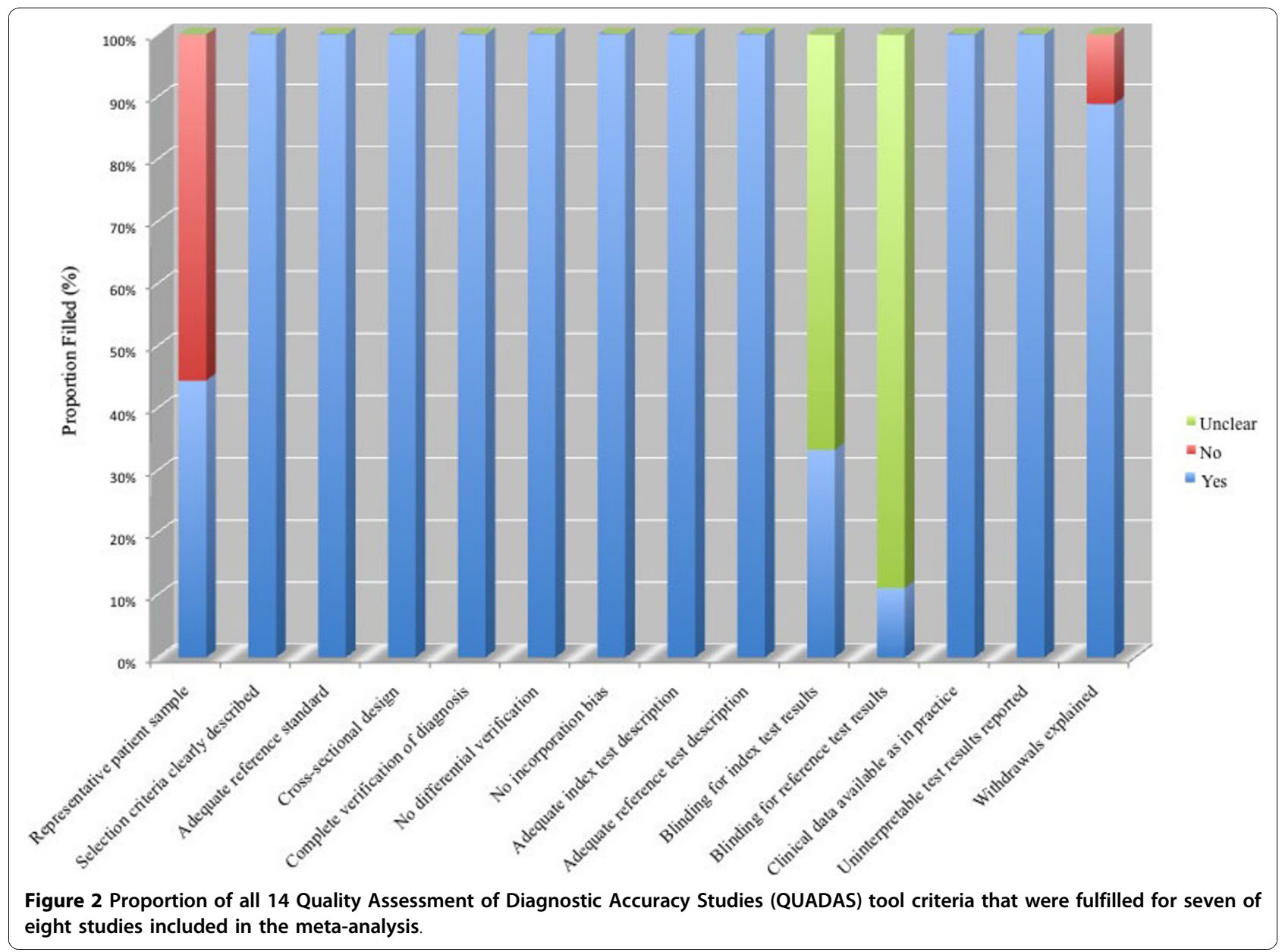

diuretics. Of the studies[18-20,23,25] that reported New York Heart Classification for the patients with effusions of cardiac origin, all patients were either class III or IV.

Overall, the quality of the reported studies was good (Figure 2). Five studies[16,19,20,23,25] maybe subject to spectrum bias as their method of recruitment of patients consisted of recruiting a target group (patients with pleural effusions of cardiac origin) and a control group (patients with pleural effusions of non-cardiac origin) rather than applying the index and reference test to an unselected patient population with pleural effusions. Most studies may be subject to review bias as it was unclear in eight studies whether the investigators who used the reference test (clinical criteria) were blinded to the results of the NT-pro-BNP assay and six studies $[16,18,20,21,24,25]$ did not state whether the laboratory personnel performing the index test (NT-pro-BNP assay) were blinded to the clinical diagnosis.

The average NT-pro-BNP level for nine of the ten studies was $6140 \mathrm{pg} / \mathrm{mL}$ in pleural effusions of cardiac origin (this value was not available for one study[22]). Of the five stu$\operatorname{dies}[16,18,19,21,24]$ that analyzed both serum and pleural fluid NT-pro-BNP levels, all five studies showed high correlation between these two parameters (Table 2). Table 2 also shows that the percentage of cardiac effusions misclassified as exudates by Light's criteria was relatively modest (range $7 \%-36 \%$ ) within various studies. However, when these misclassified effusions from Light's criteria were analyzed by NT-pro-BNP levels, the absolute number of misclassified effusions was significantly reduced (Table 2 ).

The pooled sensitivity and specificity of all studies combined was $94 \%$ (95\% CI: 90-97) and 94\% (95\% CI: 89-97) respectively (Figure 3 ). The pooled positive likelihood ratio was 15.2 (95\% CI: 8.1-28.7) and the pooled negative likelihood ratio was 0.06 (95\% CI: 0.03-0.11). The area under the ROC curve was 0.98 (95\% CI: 0.96$0.99)$ and the diagnostic odds ratio was 246 (95\% CI: 81-745). Figure 4 shows the summary receiver operating characteristic graph with $95 \%$ confidence region and 95\% prediction region for NT-pro-BNP.

The between study variability (i.e. heterogeneity) beyond what could be expected by sampling error was moderate with an $I^{2}$ of $40 \%(\mathrm{p}=0.09)$ for the sensitivity results and high with an $I^{2}$ of $84 \%(\mathrm{p}<0.01)$ for the 
Table 2 Diagnostic accuracy variables of the studies included in the meta-analysis.

\begin{tabular}{|c|c|c|c|c|c|c|c|c|c|c|c|c|}
\hline \multirow[t]{2}{*}{ Study } & \multirow{2}{*}{$\begin{array}{l}\text { No. } \\
\text { Pts/ } \\
\text { Pes }\end{array}$} & \multirow[t]{2}{*}{ TP } & \multirow[t]{2}{*}{$\mathrm{FN}$} & \multirow[t]{2}{*}{ TN } & \multirow[t]{2}{*}{ FP } & \multirow[t]{2}{*}{ Se } & \multirow[t]{2}{*}{ Sp } & \multirow{2}{*}{$\begin{array}{l}\text { NT-pro-BNP } \\
\text { Threshold* }\end{array}$} & \multirow{2}{*}{$\begin{array}{c}\text { Average } \\
\text { NT-pro- } \\
\text { BNP }^{\dagger}\end{array}$} & \multicolumn{2}{|c|}{ Misclassification } & \multirow{2}{*}{$\begin{array}{c}\text { Correlation } \\
\text { Statistic }^{\pi}\end{array}$} \\
\hline & & & & & & & & & & Light's ${ }^{\ddagger}$ & $\begin{array}{l}\text { NT-pro- } \\
\text { BNP\$\$ }\end{array}$ & \\
\hline $\begin{array}{c}\text { Tomcsanyi et al } \\
{[16]}\end{array}$ & 28 & 14 & 0 & 14 & 0 & $100 \%$ & $100 \%$ & $\geq 599^{\circ}$ & 8236 & $\begin{array}{l}1 / 14 \\
(7 \%)\end{array}$ & $0 / 1$ & $\begin{array}{l}R^{2}=0.95 \\
p<0.0001\end{array}$ \\
\hline Porcel et al[17] & 117 & 40 & 4 & 68 & 5 & $91 \%$ & $93 \%$ & $\geq 1500$ & 6931 & $\begin{array}{l}10 / 35 \\
(29 \%)\end{array}$ & $2 / 10$ & NR \\
\hline Kolditz et al[18] & 93 & 23 & 2 & 63 & 5 & $92 \%$ & $93 \%$ & $\geq 4000$ & 10427 & $\begin{array}{c}9 / 25 \\
(36 \%)\end{array}$ & $0 / 9$ & $\begin{array}{l}S=0.96 \\
p<0.001\end{array}$ \\
\hline Porcel et al[19] & 93 & 49 & 4 & 35 & 5 & $92 \%$ & $87 \%$ & $\geq 1500$ & 6106 & $\begin{array}{l}8 / 53 \\
(15 \%)\end{array}$ & $2 / 8$ & $\begin{array}{l}S=0.96 \\
p<0.001\end{array}$ \\
\hline Liao et al[20] & 40 & 10 & 0 & 29 & 1 & $100 \%$ & $97 \%$ & $\geq 2220$ & 5390 & $N R$ & NR & $N R$ \\
\hline Han et al[21] & 240 & 81 & 1 & 156 & 2 & $99 \%$ & $99 \%$ & $\geq 1714$ & 3310 & $\begin{array}{l}28 / 82 \\
(34 \%)\end{array}$ & $1 / 28$ & $\begin{array}{l}R^{2}=0.93 \\
p<0.001\end{array}$ \\
\hline Seyhan et al[22] & 115 & 47 & 4 & 61 & 3 & $92 \%$ & $95 \%$ & $\geq 1092$ & $\mathrm{~N} / \mathrm{A}$ & $\begin{array}{l}17 / 51 \\
(33 \%)\end{array}$ & N/A & N/A \\
\hline Porcel et al[23] & 181 & 84 & 6 & 81 & 10 & $93 \%$ & $89 \%$ & $\geq 1500$ & 6203 & $\begin{array}{l}20 / 90 \\
(22 \%)\end{array}$ & $4 / 20$ & NR \\
\hline Bayram et al[24] & 133 & 48 & 3 & 78 & 4 & $94 \%$ & $95 \%$ & $\geq 925$ & 4468 & $\begin{array}{l}19 / 51 \\
(37 \%)\end{array}$ & $0 / 19$ & $\begin{array}{l}R^{2}=0.91 \\
p<0.001\end{array}$ \\
\hline Long et al[25] & 80 & 16 & 4 & 44 & 16 & $80 \%$ & $73 \%$ & $\geq 2000$ & 4189 & $N R$ & NR & NR \\
\hline
\end{tabular}

* = pleural fluid NT-pro-BNP threshold in $\mathrm{pg} / \mathrm{mL} ; \dagger=$ average pleural fluid NT-pro-BNP in CHF effusions in pg/mL; $\neq=\mathrm{misclassification}$ of $\mathrm{CHF}$ effusions as exudates by Light's criteria; $\S=$ misclassification of the CHF effusions already misclassified by Light's criteria now misclassified by NT-pro-BNP; $\Pi=$ correlation between serum and pleural fluid NT-pro-BNP levels; $\uparrow=$ threshold from 599 to $1457 \mathrm{pg} / \mathrm{mL}$ resulted in $100 \%$ sensitivity and specificity; N/A = not available because only abstract available for data extraction; No. = number; NR = not recorded; Pes = pleural effusions; Pts = patients; $R^{2}=$ Pearson coefficient of correlation; S = Spearman's coefficient of rank correlation; Se = sensitivity; Sp = specificity.

specificity results. The bivariate model analysis reveals that this heterogeneity is completely (100\%) explained by threshold effect. Analysis of small study effects, potentially a result of publication bias, yielded no significant evidence for such effects with a p value of 0.26 and a funnel plot that was fairly symmetrical (Figure 5). Figure 6 illustrates the post-test probabilities based on various pre-test probabilities using a Fagan nomogram.

\section{Discussion and Conclusion}

Pleural effusions are relatively common in medical practice. It is estimated that the annual incidence of pleural effusions in the United States (US) is 1.5 million cases [26]. The most common cause of pleural effusions is CHF with an estimated incidence of 500,000 cases in the US per year[2]. Identifying the underlying etiology of pleural effusions requires a combination of strategies including clinical history, pleural fluid analysis, and potentially more invasive procedures such as pleural tissue biopsy. Clinical history is very important in the diagnosis of pleural effusions from a cardiac origin. However, alone, it does not appear to be very accurate. Romero-Candeira et al[27] studied 64 patients with transudative pleural effusions of which 44 were due to CHF and showed that in $40 \%$ of cases initial clinical history failed to correctly classify the effusion. Pleural fluid analysis using Light's criteria is better than clinical history for diagnosing pleural effusions of cardiac origin however these criteria misclassify transudates as exudative effusions approximately $25 \%$ of the time, mainly related to the use of diuretics[2]. Misclassification of transudates to exudates can lead to inappropriate management or potentially more invasive diagnostic investigations resulting in increased morbidity and health care costs. As a result, tests with higher diagnostic accuracy may prove beneficial in terms of reducing morbidity and improving cost-efficiency for diagnosing pleural effusions of cardiac origin.

In the last decade several studies have been reported assessing the diagnostic utility of pleural fluid NT-proBNP in pleural effusions of cardiac origin. To summarize these studies, we conducted a systematic review and meta-analysis. We included ten studies, of which seven were from Europe, with a total of 1120 patients and/or pleural effusions. We showed that the pooled sensitivity and specificity were identical (94\% [95\% CI: 90-97] and 94\% [95\% CI: 89-97]) with a diagnostic odds ratio of 246 (95\% CI: 81-745). When the study by Seyhan et al [22], which was retracted from the literature, was excluded from the analysis, the sensitivity, specificity, and diagnostic odds ratio remained the same within 95\% confidence intervals.

The quality of the studies was generally good overall. The main limitations of the studies were the possibility 


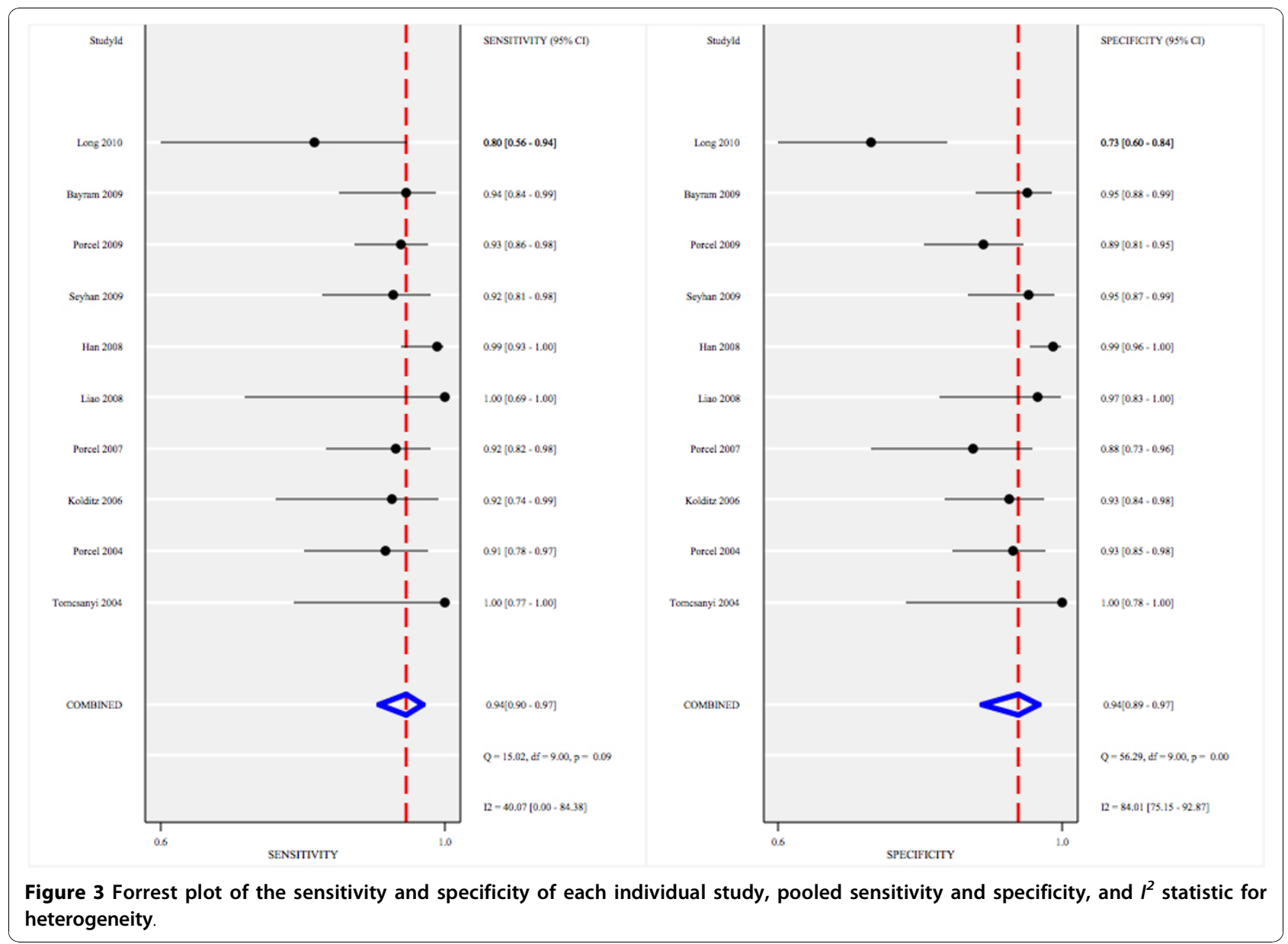

of spectrum bias, review bias, and population bias. Spectrum bias refers to distortions in a diagnostic test's performance caused by a distortion in the study population. Testing is not done across a population with the expected distribution of disease severity, but rather limited subsets[28]. In our meta-analysis, five studies $[16,19,20,23,25]$ acquired their study population by selecting CHF patients and patients with pleural effusions of non-cardiac origin (control group). The presence of spectrum bias can lead to an overestimation of the sensitivity and specificity of the test[28]. Review bias refers to a situation where persons interpreting the index test have knowledge of the reference standard or vice versa, when persons interpreting the reference standard have knowledge of the index test[29]. In our metaanalysis, it was very unclear whether this did or did not occur because the majority of the studies did not report whether blinding during testing was done. Again, this may have lead to an overestimation of the diagnostic performance of the test. Finally, population bias refers to the generalizability of the diagnostic test to a wider population[30]. The studies included in our metaanalysis had poor reporting of any co-morbid conditions within the CHF-associated pleural effusion group thus potentially limiting generalizability. Furthermore, a thoracentesis in patients with a high pre-test probability for a cardiac origin of the pleural effusion with low probability for other causes would not usually be indicated in most clinical scenarios thus further limiting the generalizability of these results.

Heterogeneity was moderate for the sensitivity results and high for the specificity results (Figure 3 ). In the summary receiver operating characteristic graph (Figure 4 ), all but three of the ten 'sensitivity - (1-specificity)' combinations of the individual studies lie on or near the summary receiver operating characteristic curve. This indicates that studies with a higher sensitivity have lower specificity and vice versa. This pattern is commonly attributed to differences in the threshold level for test positivity used in different studies[12]. As a result, the heterogeneity between the studies could well be explained by threshold level differences between the studies which ranged from 599 to $4000 \mathrm{pg} / \mathrm{mL}$. Another potential cause for the heterogeneity may be due to the various types of clinical criteria used for the reference standard (Table 1) as there is no gold standard for the 


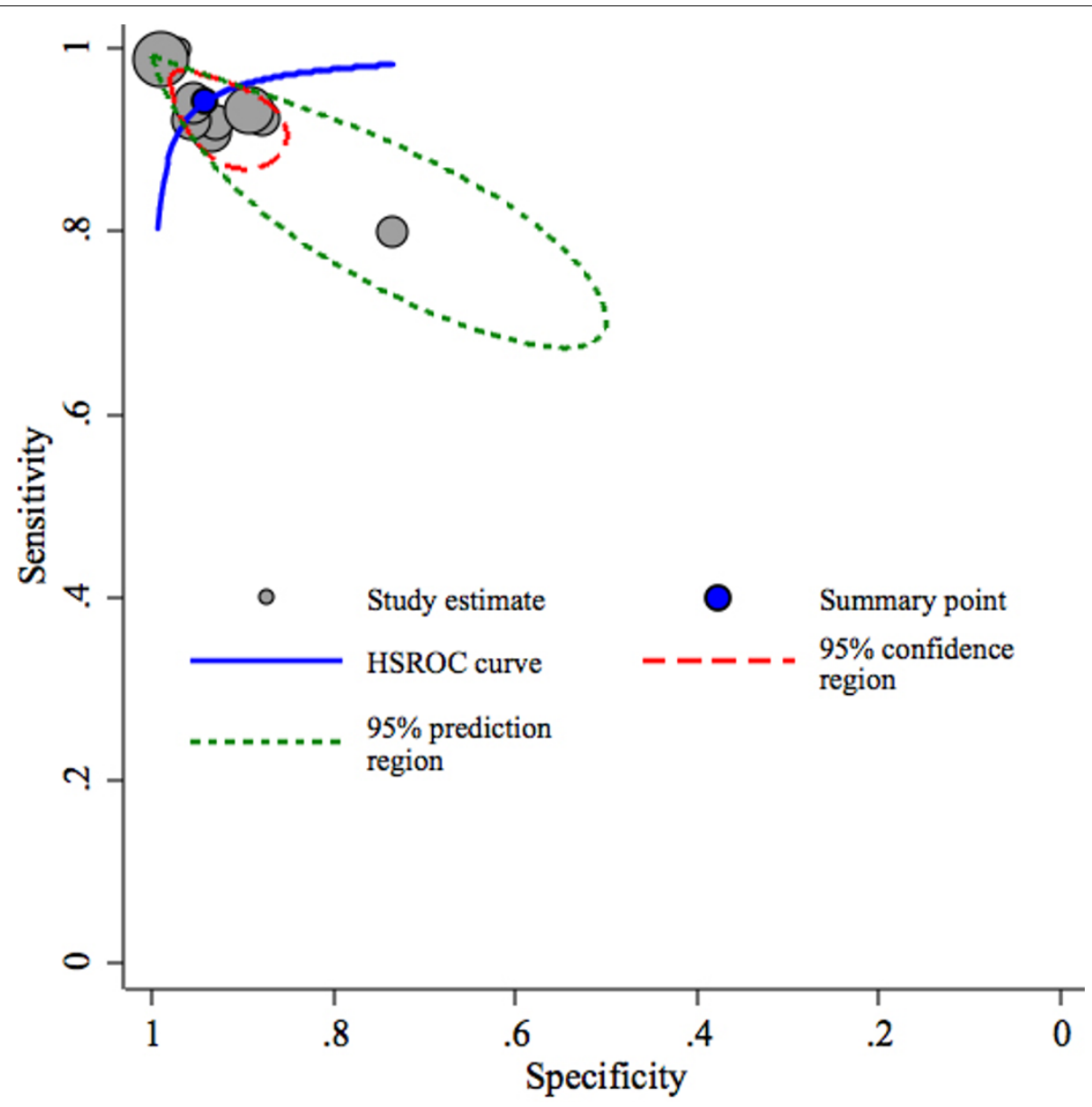

Figure 4 Summary receiver operating characteristic graph with $95 \%$ confidence region and $95 \%$ prediction region for NT-pro-BNP.

diagnosis of pleural effusions from a cardiac origin. The most objective criteria for heart failure is echocardiography and all studies except for Seyhan et al[22] used this parameter and of those studies, the majority defined $\mathrm{CHF}$ as an ejection fraction $\leq 40 \%$. Furthermore, of the studies that reported New York Heart Class, the CHFassociated pleural effusion patients were of New York Heart Class (NYHC) III or IV. The control group among the various studies differed and potentially could have contributed to the heterogeneity of the results as well. Malignant pleural effusions and parapneumonic effusions were the main groups that comprised the control groups but some studies did have other causes as a majority of the control group such as connective tissue disease, pleuritis, post-CABG, hepatic hydrothorax, and pulmonary embolism. Finally, eight of the ten studies used electrochemical luminescence immunoassay (ECLIA) for measuring NT-pro-BNP (8 studies generation one, one study generation two, one study unknown generation) while two studies used ELISA which may have added to the heterogeneity of results as well.
ECLIA uses a non-competitive immunoassay that produces luminescence via an electrochemical reaction and compared to ELISA, is faster and more precise[31]. The ELISA kit from Biomedica has been compared to the ECLIA kit from Roche Diagnostics revealing significant disparity of results between the two types of assays[31]. The Roche Diagnostics assay (ECLIA) has dual antibodies targeting two different areas of the NT-pro-BNP molecule whereas the Biomedica assay (ELISA) only has a single antibody targeting one region. This may result in better recognition of NT-pro-BNP and hence better diagnostic accuracy for the Roche Diagnostics assay. Furthermore, the cut-off values for serum NT-pro-BNP are 10 fold higher using the Biomedica assay (ELISA) compared to the Roche Diagnostics assay (ECLIA)[31] which may have also added to the heterogeneity of the results.

The correlation between pleural fluid and serum levels of NT-pro-BNP are high as shown by five studies $[16,18,19,21,24]$ in our meta-analysis (Table 2 ). This suggests that thoracentesis could potentially be avoided 


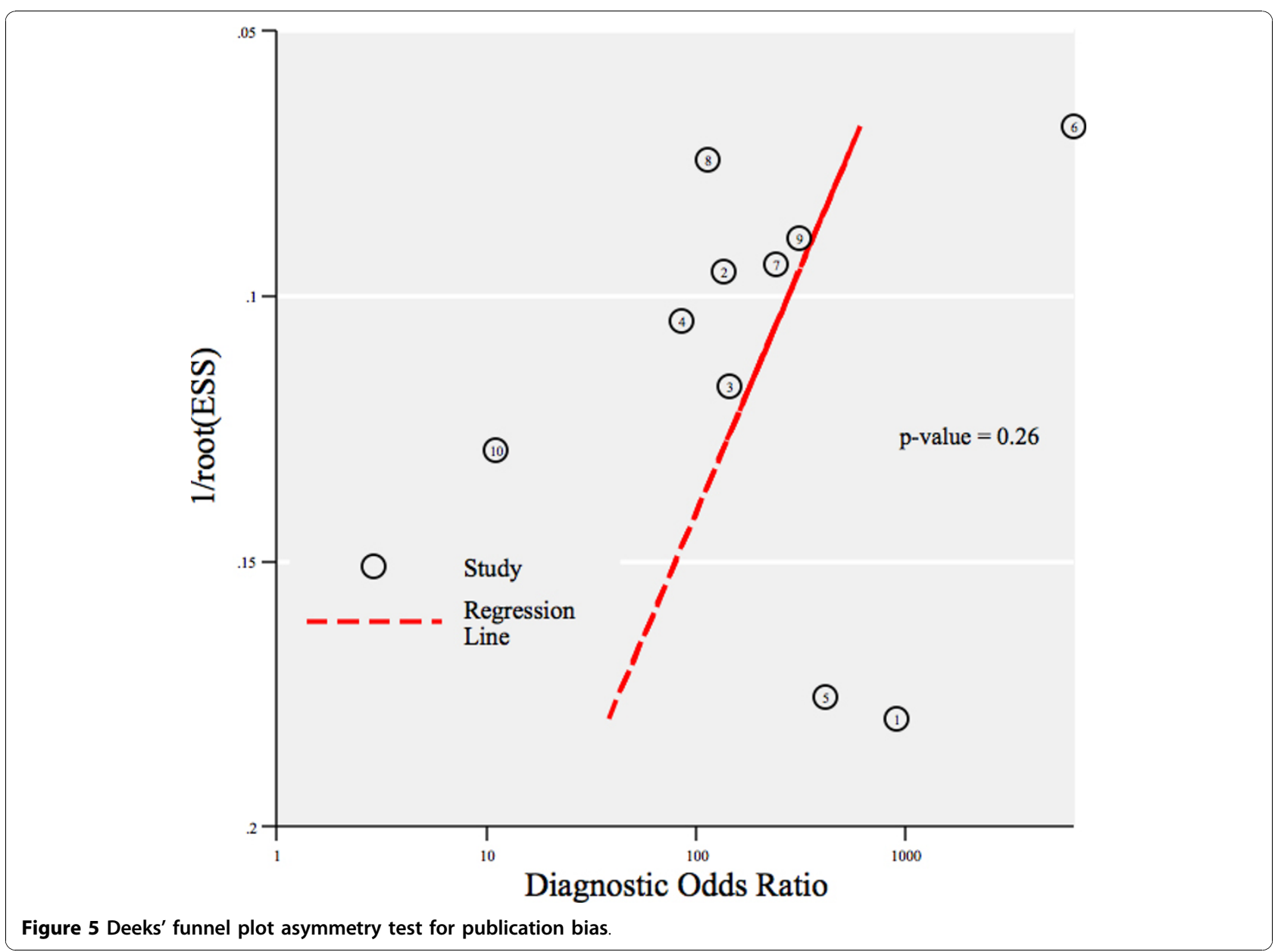

in patients with a clinical suspicion of $\mathrm{CHF}$, elevated NT-pro-BNP, and no suspicion for a co-existing cause of the pleural effusion.

Most institutions use BNP to diagnose CHF. Three studies[23,25,32] have examined the diagnostic accuracy of BNP for pleural effusions of cardiac origin. The first study[32] of 57 patients showed a sensitivity of $97 \%$ and a specificity of $89 \%$ for plasma BNP. The second study by Porcel et al[23] (181 patients), showed a sensitivity of $74 \%$ and specificity of $92 \%$ for pleural fluid BNP and also showed that the area under the curve (AUC) for NT-proBNP was higher than for pleural fluid BNP, 0.96 (95\% CI: 0.94-0.99) vs 0.90 (95\% CI: 0.86-0.95) respectively. Correlation between pleural fluid NT-pro-BNP levels and BNP levels was good, $r=0.78$ ( $p<0.001)$. The conclusion from this study was to use NT-pro-BNP rather than BNP for diagnosing pleural effusions of cardiac origin because of the diagnostic superiority of NT-pro-BNP versus BNP, greater in-vitro stability of NT-pro-BNP compared with BNP, and longer half-life of NT-pro-BNP (1-2 hours) compared with BNP (20 minutes). The last study by Long et al[25] (80 patients) showed that the AUC for
NT-pro-BNP was greater than for pleural fluid BNP, 0.84 (95\% CI: 0.72-0.95) vs 0.70 (95\% CI: 0.57-0.83). Correlation between pleural fluid NT-pro-BNP and BNP levels was also good but less impressive, $r=0.57(p<0.001)$, and only explained $32 \%$ of the variance in NT-pro-BNP. The conclusion from this study was that NT-pro-BNP was a more stable molecule after sample processing and could be maintained for a greater duration in an in vitro setting compared to BNP and furthermore because of the higher diagnostic accuracy of NT-pro-BNP, NT-pro-BNP should be used over BNP to distinguish pleural effusions of cardiac origin in select patient populations.

It is difficult to suggest a threshold level for pleural fluid NT-pro-BNP since the studies used varying threshold levels. The average pleural fluid NT-pro-BNP level in the studies was $6140 \mathrm{pg} / \mathrm{mL}$. Six of the studies $[16,17,19,21,23,24]$ used a threshold level between 1457 to $1714 \mathrm{pg} / \mathrm{mL}$ (sensitivity and specificity for a threshold level of $1457 \mathrm{pg} / \mathrm{mL}$ by ROC analysis in the study by Bayram et al[24] was $84 \%$ and $98 \%$, respectively). The pooled sensitivity and specificity of these six studies is 95\% (95\% CI: 91-98) and 95\% (95\% CI: 89-98), 


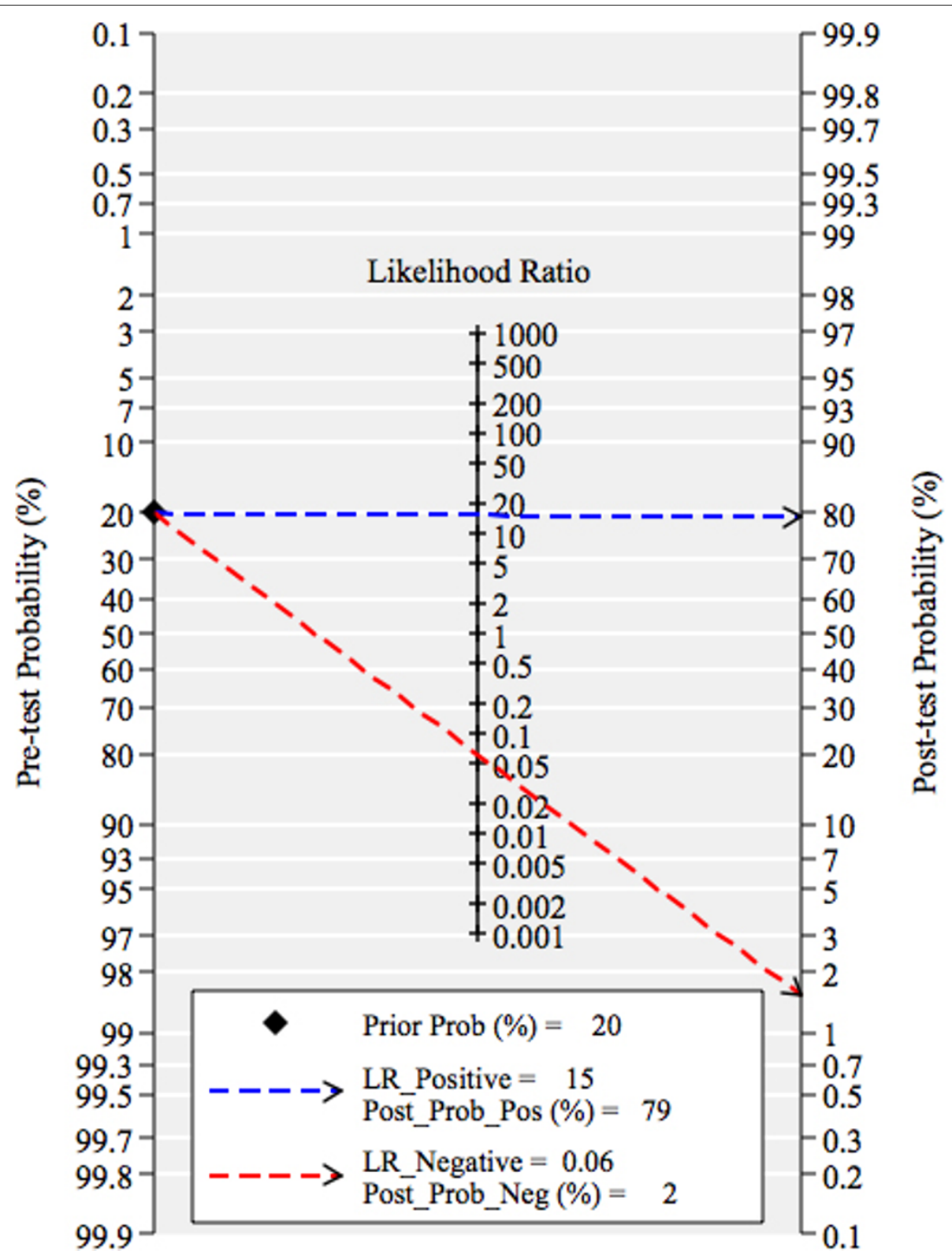

Figure 6 Fagan's nomogram for NT-pro-BNP illustrating post-test probability with a fixed pre-test probability of $20 \%$ for a pleural effusion of cardiac origin.

respectively, with a diagnostic odds ratio of 370 (95\% CI: 101-1351). As a result, a threshold level of approximately $\geq 1500 \mathrm{pg} / \mathrm{mL}$ provides very good diagnostic accuracy for pleural fluid NT-pro-BNP.

The utility of NT-pro-BNP for the diagnosis of pleural effusions of cardiac origin can be applied to several clinical situations. First, if the pre-test probability of a pleural effusion due to heart failure is high and alternative diagnoses are less likely, then a thoracentesis can be avoided and an elevated serum NT-pro-BNP level will be diagnostic for a cardiac origin of the pleural effusion. Second, if the pretest probability of a pleural effusion due to heart failure is low or alternative diagnoses are considered in addition to heart failure, then a thoracentesis should be performed and if the pleural fluid NT-pro-BNP level is high then this would be diagnostic of heart failure as one potential cause for the pleural effusion. Furthermore, in undiagnosed pleural effusions, for example when Light's criteria misclassify transudative CHF effusions as exudates and the pre-test probability is equivocal for $\mathrm{CHF}$, a high pleural fluid NT-pro-BNP would also be diagnostic.

The results of our meta-analysis are corroborated by another meta-analysis done by Zhou et al[33]. They included eight studies and found that the sensitivity and 
specificity for NT-pro-BNP for pleural effusions of cardiac origin was $95 \%$ (95\% CI: 92-97) and 94\% (95\% CI: 92-96), respectively, with a diagnostic odds ratio of 214 (95\% CI: 123-374). Based on their meta-analysis, Zhou et al[33] concluded that pleural fluid NT-pro-BNP has high diagnostic accuracy for differentiating cardiac from non-cardiac conditions in patients presenting with pleural effusions.

In summary, misclassification of pleural effusions of cardiac origin can lead to increased morbidity from further invasive testing and increased health care costs. Clinical history and pleural fluid analysis with Light's criteria may not have sufficient diagnostic accuracy to diagnose these effusions in certain clinical circumstances. Although there is some heterogeneity within this analysis, we conclude that where the diagnosis of pleural effusion of cardiac origin is under contemplation, pleural fluid NT-pro-BNP is a useful biomarker with high diagnostic accuracy.

\section{Abbreviations}

BNP: brain natriuretic peptide; CHF: congestive heart failure; ELCIA: electrochemical luminescence immunoassay; ELISA: enzyme-linked immunosorbent assay; HSROC: hierarchical summary receiver operating characteristic; NT-pro-BNP: N-terminal pro-brain natriuretic peptide; QUADAS: quality assessment of diagnostic accuracy studies; US: United States.

\section{Authors' contributions}

Both SJ and JS conducted the literature search independently. Both SJ and JS analyzed the studies to be included independently. SJ retrieved the articles. Both SJ and JS extracted the information independently. SJ conducted the statistical analysis with confirmation by JS. SJ wrote the manuscript draft. Both SJ and JS read and approved the final manuscript.

\section{Acknowledgements}

JS is a recipient of an In it for Life Scientist award from the Vancouver Coastal Health Research Institute and the Vancouver General Hospital Foundation.

\section{Competing interests}

Dr. Janda and Dr. Swiston have no competing interests.

Received: 29 August 2010 Accepted: 20 November 2010 Published: 20 November 2010

\section{References}

1. Light RW, Macgregor MI, Luchsinger PC, Ball WC Jr: Pleural effusions: the diagnostic separation of transudates and exudates. Ann Intern Med 1972, 77(4):507-513.

2. Light RW: Clinical practice. Pleural effusion. N Engl J Med 2002, 346(25):1971-1977.

3. Romero S, Candela A, Martin C, Hernandez L, Trigo C, Gil J: Evaluation of different criteria for the separation of pleural transudates from exudates. Chest 1993, 104(2):399-404

4. Roth BJ, O'Meara TF, Cragun WH: The serum-effusion albumin gradient in the evaluation of pleural effusions. Chest 1990, 98(3):546-549.

5. Tang WH, Francis GS, Morrow DA, Newby LK, Cannon CP, Jesse RL, Storrow AB, Christenson RH: National Academy of Clinical Biochemistry Laboratory Medicine Practice Guidelines: clinical utilization of cardiac biomarker testing in heart failure. Clin Biochem 2008, 41(4-5):210-221.

6. Bhalla V, Maisel AS: B-type natriuretic peptide. A biomarker for all the right reasons. Ital Heart J 2004, 5(6):417-420.
7. Porcel JM: The use of probrain natriuretic peptide in pleural fluid for the diagnosis of pleural effusions resulting from heart failure. Curr Opin Pulm Med 2005, 11(4):329-333.

8. Bhalla V, Willis S, Maisel AS: B-type natriuretic peptide: the level and the drug-partners in the diagnosis of congestive heart failure. Congest Heart Fail 2004, 10(1 Suppl 1):3-27.

9. Moher D, Liberati A, Tetzlaff J, Altman DG: Preferred reporting items for systematic reviews and meta-analyses: the PRISMA statement. Ann Intern Med 2009, 151(4):264-269, W264.

10. Whiting P, Rutjes AW, Reitsma JB, Bossuyt PM, Kleijnen J: The development of QUADAS: a tool for the quality assessment of studies of diagnostic accuracy included in systematic reviews. BMC Med Res Methodol 2003, 3:25.

11. Reitsma JB, Glas AS, Rutjes AW, Scholten RJ, Bossuyt PM, Zwinderman AH: Bivariate analysis of sensitivity and specificity produces informative summary measures in diagnostic reviews. J Clin Epidemiol 2005, 58(10):982-990.

12. Moses LE, Shapiro D, Littenberg B: Combining independent studies of a diagnostic test into a summary ROC curve: data-analytic approaches and some additional considerations. Stat Med 1993, 12(14):1293-1316.

13. Dinnes J, Deeks J, Kirby J, Roderick P: A methodological review of how heterogeneity has been examined in systematic reviews of diagnostic test accuracy. Health Technol Assess 2005, 9(12):1-113, iii.

14. Dwamena B: Midas: computational and graphical routines for metaanalytical integration of diagnostic accuracy studies in Stata. 2007.

15. Harbord R: Stata module for meta-analysis of diagnostic accuracy. 2008

16. Tomcsanyi J, Nagy E, Somloi M, Moldvay J, Bezzegh A, Bozsik B, Strausz J: NT-brain natriuretic peptide levels in pleural fluid distinguish between pleural transudates and exudates. Eur J Heart Fail 2004, 6(6):753-756.

17. Porcel JM, Vives M, Cao G, Esquerda A, Rubio M, Rivas MC: Measurement of pro-brain natriuretic peptide in pleural fluid for the diagnosis of pleural effusions due to heart failure. Am J Med 2004, 116(6):417-420.

18. Kolditz M, Halank M, Schiemanck CS, Schmeisser A, Hoffken G: High diagnostic accuracy of NT-proBNP for cardiac origin of pleural effusions. Eur Respir J 2006, 28(1):144-150

19. Porcel JM, Chorda J, Cao G, Esquerda A, Ruiz-Gonzalez A, Vives M: Comparing serum and pleural fluid pro-brain natriuretic peptide (NTproBNP) levels with pleural-to-serum albumin gradient for the identification of cardiac effusions misclassified by Light's criteria. Respirology 2007, 12(5):654-659.

20. Liao H, Na MJ, Dikensoy O, Lane KB, Randal B, Light RW: Diagnostic value of pleural fluid $\mathrm{N}$-terminal pro-brain natriuretic peptide levels in patients with cardiovascular diseases. Respirology 2008, 13(1):53-57.

21. Han $\mathrm{CH}$, Choi JE, Chung JH: Clinical utility of pleural fluid NT-pro brain natriuretic peptide (NT-proBNP) in patients with pleural effusions. Intern Med 2008, 47(19):1669-1674.

22. Seyhan EC, Altin S, Cetinkaya E, Sokucu S, Gunluoglu MZ, Demir A, Korkmaz $\mathrm{P}$, Issever $\mathrm{H}$ : The importance of pleural fluid and serum NTproBNP levels in differentiating pleural effusion due to heart failure from other causes of effusion. Intern Med 2009, 48(5):287-293.

23. Porcel JM, Martinez-Alonso M, Cao G, Bielsa S, Sopena A, Esquerda A: Biomarkers of heart failure in pleural fluid. Chest 2009, 136(3):671-677.

24. Bayram M, Ozkan G, Oztekin E, Bakan ND, Acikmese B, Bes S, Gur A, Camsari G: Role of serum and pleural fluid NT-proBNP levels in identifying pleural effusion due to heart failure. Multidisciplinary Respiratory Medicine 2009, 4(3):175-181.

25. Long AC, O'Neal HR Jr, Peng S, Lane KB, Light RW: Comparison of pleural fluid $\mathrm{N}$-terminal pro-brain natriuretic peptide and brain natriuretic-32 peptide levels. Chest 2010, 137(6):1369-1374.

26. Sahn SA: The value of pleural fluid analysis. Am J Med Sci 2008 , 335(1):7-15.

27. Romero-Candeira S, Hernandez L, Romero-Brufao S, Orts D, Fernandez C, Martin C: Is it meaningful to use biochemical parameters to discriminate between transudative and exudative pleural effusions? Chest 2002, 122(5):1524-1529.

28. Ransohoff DF, Feinstein AR: Problems of spectrum and bias in evaluating the efficacy of diagnostic tests. N Engl J Med 1978, 299(17):926-930.

29. Begg CB: Biases in the assessment of diagnostic tests. Stat Med 1987, 6(4):411-423. 
30. Kelly S, Berry E, Roderick P, Harris KM, Cullingworth J, Gathercole L, Hutton J, Smith MA: The identification of bias in studies of the diagnostic performance of imaging modalities. Br J Radiol 1997, 70(838):1028-1035.

31. Mueller T, Gegenhuber A, Poelz W, Haltmayer M: Comparison of the Biomedica NT-proBNP enzyme immunoassay and the Roche NT-proBNP chemiluminescence immunoassay: implications for the prediction of symptomatic and asymptomatic structural heart disease. Clin Chem 2003, 49(6 Pt 1):976-979.

32. Gegenhuber A, Mueller T, Dieplinger B, Lenz K, Poelz W, Haltmayer M: Plasma B-type natriuretic peptide in patients with pleural effusions: preliminary observations. Chest 2005, 128(2):1003-1009.

33. Zhou Q, Ye ZJ, Su Y, Zhang JC, Shi HZ: Diagnostic value of N-terminal pro-brain natriuretic peptide for pleural effusion due to heart failure: a meta-analysis. Heart 96(15):1207-1211.

\section{Pre-publication history}

The pre-publication history for this paper can be accessed here: http://www.biomedcentral.com/1471-2466/10/58/prepub

doi:10.1186/1471-2466-10-58

Cite this article as: Janda and Swiston: Diagnostic accuracy of pleural fluid NT-pro-BNP for pleural effusions of cardiac origin: a systematic review and meta-analysis. BMC Pulmonary Medicine 2010 10:58.

\section{Submit your next manuscript to BioMed Central} and take full advantage of:

- Convenient online submission

- Thorough peer review

- No space constraints or color figure charges

- Immediate publication on acceptance

- Inclusion in PubMed, CAS, Scopus and Google Scholar

- Research which is freely available for redistribution

Submit your manuscript at www.biomedcentral.com/submit 